\title{
Association of Basophil-Expressing Genes with Effectiveness of Sublingual Immunotherapy
}

\author{
Minoru Gotoh ${ }^{1,2, \dagger}$, Osamu Kaminuma ${ }^{2,3, \dagger}$, Akihiro Nakaya ${ }^{2,4, \dagger}$, Mayumi Saeki ${ }^{2}$, \\ Tomoe Nishimura ${ }^{2}$, Noriko Kitamura ${ }^{2}$, Takachika Hiroi ${ }^{2}$ and Kimihiro Okubo ${ }^{1,2}$ \\ ${ }^{1}$ Department of Otorhinolaryngology, Nippon Medical School, Tokyo, Japan \\ ${ }^{2}$ Allergy and Immunology Project, The Tokyo Metropolitan Institute of Medical Science, Tokyo, Japan \\ ${ }^{3}$ Department of Disease Model, Research Institute of Radiation Biology and Medicine, Hiroshima University, Hiroshima, Japan \\ ${ }^{4}$ Department of Genome Informatics, Graduate School of Medicine, Osaka University, Osaka, Japan
}

\begin{abstract}
Basophils were reported to be associated with allergy pathogenesis and the efficacy of allergen immunotherapy. Using a purified cedar allergen, we recently studied the effectiveness of sublingual immunotherapy for patients with Japanese cedar pollinosis. Patients were classified as high responders (HR) and nonresponders (NR), and comprehensive microarray analysis was used to examine peripheral basophils in both groups. A total of 153 genes were differentially expressed in HR and NR patients. Most of these differentially expressed genes encoded intracellular molecules, and expression levels were higher in HR patients than in NR patients. mRNA expression of the gene encoding D4, zinc, and double plant homeodomain (PHD) fingers family 2 (DPF2) was significantly correlated with copy number variation (CNV). Genetic variation in the DPF2 gene and its expression in basophils might be associated with the efficacy of sublingual immunotherapy. (J Nippon Med Sch 2020; 87: 49-52)
\end{abstract}

Key words: apoptosis, copy number variation, D4, zinc, and double plant homeodomain fingers family 2, microarray analysis

\section{Introduction}

A number of sublingual immunotherapy (SLIT) drugs for treating allergic rhinitis have recently been marketed in Japan. They are attractive because of their high efficacy and potential to provide a radical treatment. However, SLIT does not benefit some patients. We previously compared SLIT nonresponders (NR) and high responders (HR) and identified potential determinants of SLIT efficacy, namely, patients with high correlations among serum Th1/Th2 cytokines tended to respond well to SLIT ${ }^{1}$. An important source of Th1/Th2 cytokines is $\mathrm{CD}^{+} \mathrm{T}$ cells, and taste receptors on $\mathrm{CD}^{+} \mathrm{T}$ cells may contribute to SLIT efficacy ${ }^{2}$.

Basophils have recently served as targets for allergen immunotherapy. Basophils produce IL-4 in response to various stimuli, including IgE-dependent allergen recog- nition $^{3}$. The proportions of basophils expressing C-type lectin receptors and Fc $\gamma \mathrm{R}$ II were reduced and increased, respectively, by subcutaneous immunotherapy ${ }^{4}$. Epicutaneous immunotherapy for patients with peanut allergy downregulated CD63 expression in basophils, increased peanut-specific IgG4 and IgG4/IgE ratios, and decreased Th2 cytokine production ${ }^{5}$. Herein, we used comparative microarray mRNA expression analysis of basophils collected from HR and NR patients to identify basophildependent mechanisms that might explain SLIT efficacy.

\section{Materials and Methods \\ SLIT for Japanese Cedar Pollinosis (JCP)}

The study design, patient recruitment, administration of cedar pollen extract, and evaluation of clinical efficacy were performed as described recently ${ }^{1}$. The present study

\footnotetext{
${ }^{\dagger}$ These authors contributed equally to this work.

Correspondence to Minoru Gotoh, MD, PhD, Department of Otorhinolaryngology, Nippon Medical School, 1-1-5 Sendagi,

Bunkyo-ku, Tokyo 113-8603, Japan

E-mail: m.gotoh@nms.ac.jp

https://doi.org/10.1272/jnms.JNMS.2020_87-104

Journal Website (https://www.nms.ac.jp/sh/jnms/)
} 
included 202 adult JCP patients who presented with symptoms of allergic rhinitis from February through April for at least 3 consecutive years. The patients had positive results on a skin-test and for $\operatorname{IgE}$ against cedar pollen allergen. The study was registered in the University Hospital Medical Information Network Clinical Trials Registry Database (UMIN000016532) and was conducted in accordance with the Declaration of Helsinki and Good Clinical Practice guidelines. Experimental procedures were approved by the ethical committee of all participant institutions. All patients provided informed written consent before participation.

Cedar pollen allergen was administered to all participants, as described previously ${ }^{1}$. Starting with one drop of 2 Japanese allergy units (JAU)/mL, we gradually increased the amount of allergen until it reached a concentration of 20 drops of 2,000 JAU/mL as the maintenance dose at 5 weeks, which was continued for 2 years.

Nasal symptoms of patients from February 1st through April 30th during four pollen seasons were investigated in accordance with Japanese guidelines for allergic rhinitis. The patients recorded their symptoms in an allergy diary, as previously described ${ }^{1}$. Patient quality of life was determined thrice annually - at the end of February, in the middle of March, and in the middle of April-by using the Japan Rhinoconjunctivitis Quality of Life Questionnaire No.1 (JRQLQ No1). At the end of each pollen season, SLIT efficacy was evaluated by analyzing information from the allergy diary and JRQLQ No1 ${ }^{1}$.

\section{Cell Preparation and Microarray Analysis}

Blood samples were collected twice: immediately before the start and at the end of allergen administration. Basophils were purified as $\mathrm{CD} 123^{+} \mathrm{CD} 11 \mathrm{c}^{\text {low }} \mathrm{BDCA}-1^{-}$ BDCA-4 ${ }^{-} \mathrm{HLA}_{-} \mathrm{DR}^{-}$cells from peripheral blood, as previously described ${ }^{1}$. Genomic DNA (gDNA) of the samples was extracted from blood cells.

Microarray analysis of basophils was performed as previously described ${ }^{6}$. Comparative genome-wide transcriptome analysis was performed by using the GeneChip Human Gene 1.0 ST Array Platform (Thermo Fisher Scientific, Waltham, MA); 17,743 of 33,297 probe sets were analyzed for transcripts associated with specific functionally annotated sites in the genome sequences. The results were normalized as z-scores by using the equation $\mathrm{z}=[\mathrm{v}-\mu] / \sigma$, where $\mathrm{v}$ is the raw value for a patient, $\mu$ is the mean value for all HR and NR patients, and $\sigma$ is the associated standard deviation in the population. The $\log 2$ ratios of the averaged data were calculated between the HR and NR groups.

\section{Analysis of Copy Number Variation (CNV)}

CNV analysis was performed as previously described ${ }^{2}$. The reaction products were analyzed with an Agilent SurePrint G3 Human CNV MicroArray Kit, 2×400K, using the Japanese male HapMap sample NA19000 and UCSC Human Genome build hg19. Probes matching single gDNA sites extracted 2,959 CNV regions. Among them, CNV regions with $\geq 3$ probes and a $\log 2$ ratio of $\geq 0.25$ were identified with ADM- 2 algorithms.

\section{Statistical Analysis}

Data for mRNA analyses are presented in heat maps. Linear regression analysis was used to identify correlations between CNV and mRNA levels.

\section{Results}

Twenty-five HR and NR JCP patients were selected from patients who received 2-year SLIT, as described previously $^{1}$. RNA of post-SLIT basophils of both groups was assessed in microarray analysis. The peripheral basophil population was unaffected by SLIT, and the population did not differ between HR and NR patients, as described $^{1}$. However, we identified 153 genes that were differentially expressed between HR and NR patients (Fig. 1 A). Most of these genes were more strongly expressed in HR than in NR patients; only 12 genes had higher expression levels in NR patients. Many genes encoded intracellular molecules, such as transcription factors, enzymes, and interacting proteins. Approximately 30 small nuclear RNAs were identified (Fig. 1B).

We next investigated associations between gene expression levels and corresponding CNV values and observed a significant correlation in only one gene/CNV pair, namely, the D4, zinc, and double plant homeodomain (PHD) fingers family 2 (DPF2) (Fig. 2). Gene expression and $\mathrm{CNV}$ in the DPF2 gene were significantly correlated in the HR, NR, and HR plus NR groups.

\section{Discussion}

The number of differentially expressed genes in basophils (153 genes) in this study was larger than that (56 genes) reported in $\mathrm{CD}^{+} \mathrm{T}$ cells ${ }^{2}$. We previously evaluated the significance of genes differentially expressed in $\mathrm{CD}^{+} \mathrm{T}$ cells of HR and NR patients by analyzing their associations with CNVs. Because CNV is generated by multiplication and deletion of DNA segments of $10^{3}$ to $10^{6} \mathrm{nu}-$ cleotides, which frequently contain an entire gene with its regulatory region, $\mathrm{CNVs}$ often directly affect gene expression levels ${ }^{7}$. Expression and $\mathrm{CNV}$ were significantly correlated only in the DPF2 gene. DPF2 is a ubiquitously 
A
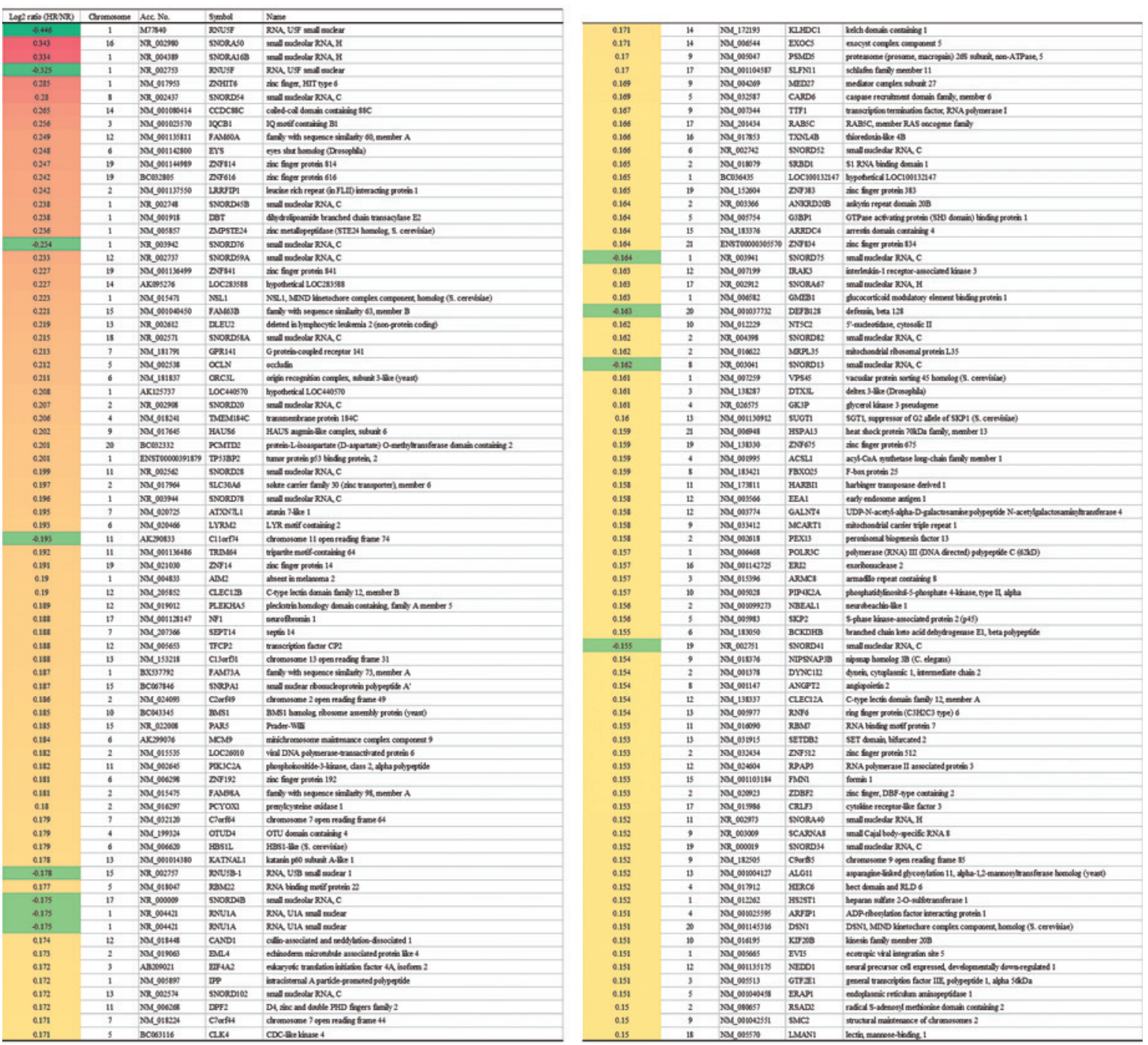

B

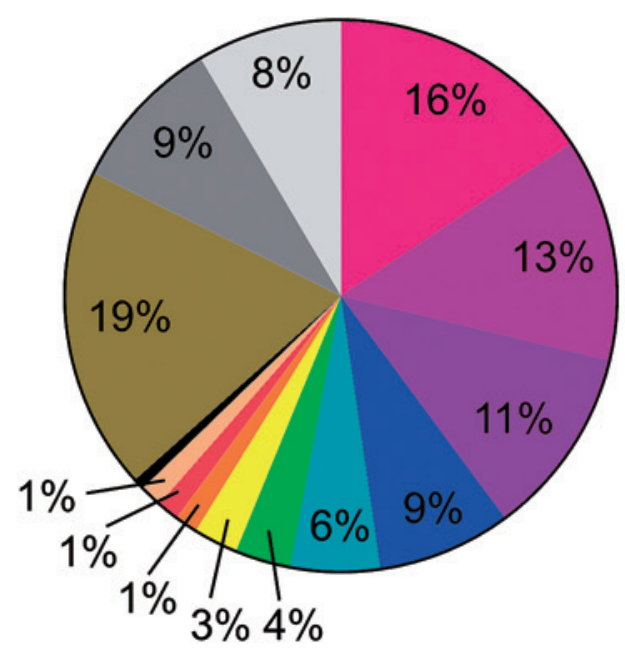

$\square$ Transcription
Enzyme
Protein interaction
Cell cycle
Membrane
Translation
$\square$ Cytoskeletal
Metabolism
$\square$ Mitochondrial
Nuclease
Soluble
$\square$ Small nuclear
Other
Unknown

Fig. 1 Differential mRNA expression in basophils from HR and NR patients. Genome-wide transcriptome analysis of basophils from HRs and NRs was performed as described in the Methods. Log2 ratios of average expression levels in basophils between HR and NR patients after SLIT (Post) are indicated in red (HR > NR) and green $(H R<N R)$. Genes with a difference in $\log 2$ ratio greater than 0.15 between HRs and NRs before and after treatment are listed (A). The classification and percentage of identified genes are shown (B). 
ALL

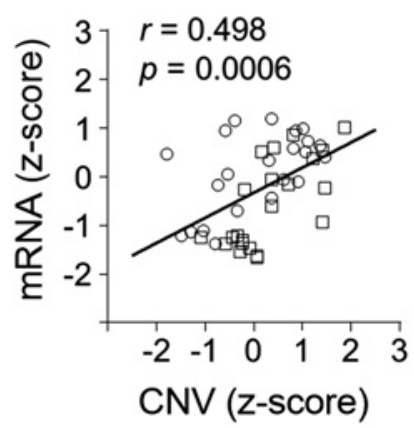

HR

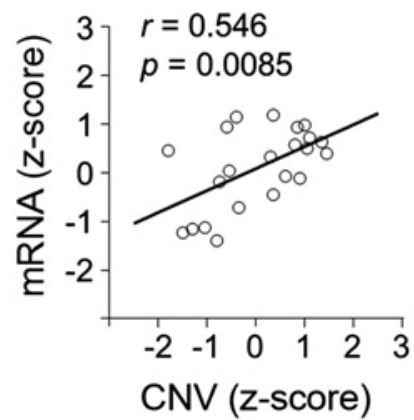

NR

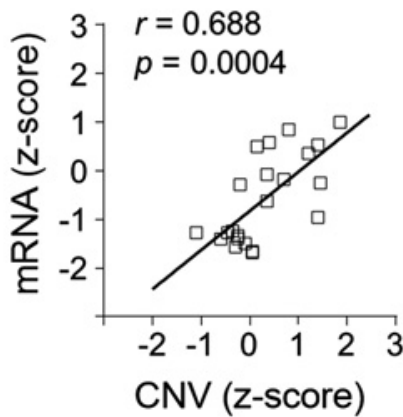

Fig. 2 Correlation between expression and CNV in the DPF2 gene. The correlations between DPF2 mRNA expression and CNV in the HR plus NR (ALL; left panel), HR (center), and NR (right) groups before SLIT were examined. Normality of the distributions was confirmed with the D'Agostino-Pearson omnibus normality test.

expressed d4-protein family member and is multifunctional $^{8,9}$. It was reported to influence gene transcription, the noncanonical NF- $\kappa$ B pathway, chromatin organization, and protein-protein interactions via its PHD domain ${ }^{8}$. Involvement of DPF2 in apoptosis ${ }^{9}$ is consistent with our previous findings, which suggested that the apoptotic pathway in basophils contributes to SLIT efficacy $^{6}$. Future studies should examine the role of DPF2 in basophil function.

The significant correlation between expression and CNV in the DPF2 gene was similar across the HR, NR, and HR plus NR groups. These results are inconsistent with our previous finding of a substantial correlation between TAS2R43 gene expression in $\mathrm{CD}^{+} \mathrm{T}$ cells and our observation of CNV in HR but not NR patients ${ }^{2}$. The reason for these differing correlation patterns is unclear, although distinct mechanisms may be involved. The correlated and uncorrelated patterns in HR and NR patients, respectively, in the TAS2R43 genes suggest that some factor other than its CNV regulates TAS2R43 expression in $\mathrm{CD}^{+} \mathrm{T}$ cells in NR patients. In contrast, $\mathrm{CNV}$ in the DPF2 gene, which affects its expression in basophils from both HR and NR patients, could act as a direct determinant of SLIT efficacy.

In conclusion, we identified genes differentially expressed in basophils from HR and NR patients. DPF2 expression was correlated with its CNV. Future studies should compare HR patients before and after SLIT, and functional studies of basophils could help identify the mechanisms related to SLIT efficacy.

Acknowledgements: This work was supported by a grant-inaid for scientific research from the Japan Society for the Promotion of Science (KAKENHI, 15K08626 and 19K09901 to
M.G.) and by funding from the Kurozumi Medical Foundation, Japan Allergy Foundation, Shimabara Science Foundation, and Suzuken Memorial Foundation (to O.K.).

Conflict of Interest: The authors declare no conflicts of interest.

\section{References}

1. Gotoh M, Kaminuma O, Nakaya A, et al. Identification of biomarker sets for predicting the efficacy of sublingual immunotherapy against pollen-induced allergic rhinitis. Int Immunol. 2017;29:291-300.

2. Gotoh $\mathrm{M}$, Kaminuma $\mathrm{O}$, Nakaya $\mathrm{A}$, et al. Involvement of taste receptors in the effectiveness of sublingual immunotherapy. Allergol Int. 2018;67:421-4.

3. Miyake K, Karasuyama H. Emerging roles of basophils in allergic inflammation. Allergol Int. 2017;66:382-91.

4. Lundberg K, Rydnert F, Broos S, Andersson M, Greiff L, Lindstedt M. C-type Lectin Receptor expression on human basophils and effects of allergen-Specific immunotherapy. Scand J Immunol. 2016;84:150-7.

5. Jones SM, Sicherer SH, Burks AW, et al. Epicutaneous immunotherapy for the treatment of peanut allergy in children and young adults. J Allergy Clin Immunol. 2017;139: 1242-1252.e9.

6. Gotoh M, Kaminuma O, Hiroi T, Okubo K. Microarraybased multivariate analysis of the effectiveness of sublingual immunotherapy for cedar pollinosis. Allergy Asthma Immunol Res. 2018;10:562-9.

7. Riggs ER, Ledbetter DH, Martin CL. Genomic Variation: lessons learned from whole-genome CNV analysis. Curr Genet Med Rep. 2014;2:146-50.

8. Liu C, Zhang D, Shen Y, et al. DPF2 regulates OCT4 protein level and nuclear distribution. Biochim Biophys Acta. 2015;1853:3279-93.

9. Gabig TG, Mantel PL, Rosli R, Crean CD. Requiem: a novel zinc finger gene essential for apoptosis in myeloid cells. J Biol Chem. 1994;269:29515-9.

(Received,

July 4, 2019)

(Accepted,

August 23, 2019)

(J-STAGE Advance Publication, September 30, 2019) 